\title{
Hiperlaxitud articular en población femenina que labora como personal administrativo y que es atendido en un Centro de terapia física
}

Joint hypermobility in a female population who works as administrative personnel and who is cared for in a physical therapy center

\author{
Adriana Inés Nicho Barrera ${ }^{1}$, Stephany Fabiola Pozo Guerrero ${ }^{1}$, Paul Rubén Alfaro Fernández ${ }^{2}$, Elizabeth \\ Cecilia Meléndez Olivari ${ }^{1}$
}

\section{RESUMEN}

La hiperlaxitud articular afecta a más del $10 \%$ de la población presentándose con mayor frecuencia en mujeres y en niños. Objetivo: Determinar la frecuencia de hiperlaxitud articular en población femenina que labora como personal administrativo y que asiste para tratamiento fisioterapéutico o evaluación ocupacional a un centro de terapia física en el distrito de Comas. Material y métodos: Estudio de tipo descriptivo, observacional tipo transversal, no probabilístico. La población estudiada fue 153 mujeres que laboran en áreas administrativas y que asistieron para tratamiento fisioterapéutico o evaluación ocupacional a un centro de terapia física en el distrito de Comas. Para la recolección de datos se utilizó una ficha de datos y el test de Beighton para evaluar la hiperlaxitud articular. Resultados: Se obtuvo como resultado que el $42,5 \%$ de esta población presentaba hiperlaxitud articular, IC $95 \%(34,6 \%-50,4 \%)$ y que su frecuencia va disminuyendo en edades más adultas. Las articulaciones con mayor frecuencia de hiperlaxitud articular son: meñique derecho $(54,90 \%)$, meñique izquierdo $(45,10 \%)$ y codo derecho (45,10\%). Conclusiones: Se concluyó que la frecuencia de hiperlaxitud articular en mujeres que laboran en áreas administrativas es del $42,5 \%$ y que disminuye conforme avanza la edad, datos similares al de otros estudios.

PALABRAS CLAVE: Hiperlaxitud articular, hipermovilidad articular, estrés ocupacional, personal administrativo.

\section{SUMMARY}

Joint hypermobility affects more than $10 \%$ of the population, occurring more frequently in women and children. Objective: To determine the frequency of joint hypermobility in the female population that works as administrative personnel and that attends a physical therapy center in the district of Comas for physiotherapeutic treatment or occupational evaluation. Material and methods: Descriptive, observational, cross-sectional, non-probabilistic study. The studied population was 153 women who work in administrative areas and who attended a physical therapy center in the district of Comas for physiotherapeutic treatment or occupational evaluation. For data collection, a data sheet and the Beighton test were used to assess joint hypermobility. Results: It was obtained as a result that $42.5 \%$ of this population presented joint hypermobility, $95 \%$ CI $(34.6 \%-50.4 \%)$ and that its frequency decreases in older ages. The joints with the highest frequency of joint hypermobility are: right little finger (54.90\%), left little finger $(45.10 \%)$ and right elbow (45.10\%). Conclusions: It was concluded that the frequency of joint hypermobility in women who work in administrative areas is $42.5 \%$ and that decreases with age, data similar to those of other studies.

KEY WORDS: Joint hypermobility, joint hypermobility, occupational stress, administrative staff.

\footnotetext{
Escuela de Tecnología Médica, Facultad de Medicina, Universidad Peruana Cayetano Heredia. Lima, Perú
}

2 Universidad de San Martín de Porres. Lima, Perú 


\section{INTRODUCCIÓN}

La hiperlaxitud articular (HA) se refiere al incremento exagerado de la movilidad articular, es decir, el rango de movimiento supera los límites normales $(1,2)$. Esta condición se ve influenciada por el sexo, la edad y los antecedentes étnicos del individuo $(3,4)$. Según datos, en los países europeos la HA afecta a más del 10\% de la población, en el caso de los países occidentales, es el 15\%, y en otras poblaciones llega hasta el 25\% presentándose con mayor frecuencia en mujeres que en hombres y en niños más que en adultos $(1,3)$. En el caso de la mujer, esto podría deberse a la influencia hormonal, principalmente de la relaxina, la cual actúa indirectamente sobre los ligamentos brindando mayor capacidad de elasticidad y por ende mayor movilidad a las estructuras (5).

Su etiología es desconocida, sin embargo, se indica que la HA se presenta a nivel de ligamentos, tendones, vasos sanguíneos y piel, generando en ellos, una elasticidad más allá de lo habitual y predisponiéndolos a sufrir lesiones frecuentes (1). Algunos autores también indican que la causa principal de HA es la laxitud ligamentosa determinado por genes de las proteínas fibrosas (5), de ahí, que las personas hiperlaxas poseen mayor cantidad de elastina con relación al colágeno en los ligamentos, tendones y músculos, volviéndolos frágiles. La hiperlaxitud articular se caracteriza por no presentar sintomatología, y de presentarse, se le conocerá como Síndrome de Hiperlaxitud Articular (3).

Por otro lado, sabemos que el personal administrativo es un grupo laboral que se encarga de realizar tareas propias de oficina tales como redacción y/o confección de cartas comerciales, recepción y clasificación de correspondencia, archivo de documentación, mantención de registros de personal u otros (6), todas estas actividades suelen desarrollarse en posiciones constantes, movimientos repetitivos principalmente en miembros superiores y de largas horas, lo que conllevaría a la fatiga y stress articular. En nuestro país, esta población ocupa un porcentaje relevante con relación a otros grupos laborales, es así como, en un estudio del año 2014 realizado por el Instituto Nacional de Estadística e Informática donde se encuestó a 10564 egresados de universidades públicas y privadas, se encontró que el 16,5\% laboraba en alguna de las áreas de las Ciencias administrativas y contables (7). Strazdins y Bammer, realizaron un estudio de comparación de trabajo estático entre hombres y mujeres en Australia, donde se efectúo una encuesta a 737 empleados de servicio público, obteniendo que el $34 \%$ de mujeres se mantienen sentadas en la misma posición por largos periodos de tiempo en comparación con el $21 \%$ de hombres, el $81 \%$ de mujeres trabajan 5 horas por día en el ordenador en comparación con el $73 \%$ de hombres, y el 30\% de mujeres informaron que su trabajo consistía en la realización de movimientos repetitivos en comparación con el 16\% de hombres (8). Por lo tanto, no es difícil pensar que la condición de HA sumado a la actividad laboral administrativa, conlleve a estas personas a una condición más vulnerable de lesión, afectando su calidad de vida en su presente o futuro. Si a ello, se añade el factor sexo, siendo en su mayoría población femenina, el impacto en la salud es mayor. También podemos observar que en la norma básica de ergonomía y de procedimiento de evaluación de riesgo disergonómico, se exponen ciertas pautas para la identificación de los factores de riesgo disergonómicos, los cuales controlado a tiempo podrían reducir la incidencia y severidad de los disturbios musculo esqueléticos que se pueden generar relacionados con este tipo de trabajo (9).

Al no hallar datos ni estudios a nivel nacional que proporcionen información de HA en esta población, las investigadoras consideramos importante realizar este estudio que servirá como estudio base para otras investigaciones relacionadas al área de la ergonomía y salud de la mujer. El objetivo principal del estudio fue determinar la frecuencia de HA en el personal administrativo femenino que labora como personal administrativo y que asiste para tratamiento fisioterapéutico o evaluación ocupacional a un centro de terapia física en el distrito de Comas, junto con la frecuencia de HA con relación a la edad y sexo, y explorar la relación entre HA, jornada laboral y actividad laboral.

\section{MATERIAL Y MÉTODOS}

El diseño de estudio fue de tipo descriptivo, observacional, transversal, no probabilístico. La población de estudio fue el personal femenino que laboraba en áreas administrativas y que asistían a un centro de terapia física en el distrito de Comas para tratamiento fisioterapéutico o evaluación ocupacional. Esta selección se hizo en base a los descrito en la literatura que nos señala la tendencia que tiene el sexo femenino a presentar con mayor frecuencia Hiperlaxitud articular. Los criterios de inclusión considerados fueron: mujeres en edades entre $20 \mathrm{y}$ 65 años que asisten al centro de terapia física y que, 
mediante el consentimiento informado, autorizaron su evaluación para el estudio. En los de exclusión se consideró: mujeres que hayan tenido fracturas no consolidadas, presencia de dolor articular, mujeres embarazadas y mujeres que no autoricen su evaluación. El tamaño de muestra se obtuvo considerando una frecuencia esperada del $10 \%$ basándonos en el estudio de Bravo (4), con un margen de error de 0,05, dando como resultado una muestra de 139 personas según el programa GRANMO. Considerando un $10 \%$ de pérdidas, el tamaño final de muestra para este estudio fue de 154 personas. La técnica de muestreo fue no probabilística, por conveniencia basándose en el enrolamiento consecutivo de todas las personas que aceptaron participar. Se enrolaron participantes hasta completar el tamaño de muestra estipulado en esta investigación.

En cuanto a los procedimientos y técnicas, se incluyó al personal administrativo femenino que asistió a un centro de terapia física en el distrito de Comas a la cual se le solicitó la firma correspondiente en la ficha de consentimiento informado. Las investigadoras fueron previamente capacitadas en el uso del test de Beighton. Se aplicó la prueba de Kappa de Cohen para la evaluación de la concordancia que existe en la aplicación de esta prueba piloto, dando un valor de concordancia del $79.4 \%$ siendo este porcentaje bueno (60-80\%) para la ejecución de este estudio. El instrumento utilizado fue la prueba de Beighton, el cual evalúa la presencia o ausencia de hiperlaxitud articular con un puntaje de 0 a 9 siendo a partir del puntaje 4 positivo para la hiperlaxitud articular. Para ello se ejecutaron las maniobras descritas por Beighton, Solomo, y Soskolne en su publicación titulada "Articular mobility in an African population" (10). Para la medición de los rangos articulares en el caso del dedo, codo y rodilla se utilizó como instrumento el goniómetro. La evaluación se ejecutó en un centro de terapia física en el distrito de Comas cuya duración fue de 15 minutos por persona aproximadamente. La afluencia de personas que asistían al centro de terapia física fue de 36 de los cuales 2 o 3 cumplían con los criterios de inclusión por lo cual fue necesario 2 meses para completar el tamaño de muestra estipulado. Para el análisis descriptivo las variables continuas fueron presentadas a través del promedio y desviación estándar, y las variables categóricas fueron presentadas a través del porcentaje. Se usó tablas de doble entrada para mostrar los resultados y para el análisis estadístico se utilizó el Software Stata versión 12.

\section{RESULTADOS}

Del total de la muestra, $153(99,35 \%)$ participaron en el estudio. La frecuencia de hiperlaxitud articular fue 42,5\%; con el intervalo de confianza (IC) al 95\%, tenemos que la verdadera proporción esta entre el $34,6 \%$ y el $50,4 \%$. Se encontró que la edad media fue de 37,1 años con desviación estándar de 8,7 (rango 20 - 55años). Sobre la jornada laboral se encontró que el $33.3 \%$ laboraba 8 horas, el $25,5 \% 9$ horas y el $41,2 \%$ 10 horas. De las actividades laborales que realizaban se encuentra que el $55,6 \%$ se desempeñaban como ordenadores, el $34,6 \%$ en la recepción y el $9,8 \%$ en archivo. Como características de las evaluadas también

Tabla 1. Características del personal administrativo

\begin{tabular}{lcc}
\hline Promedio de edad (años) +/- DS & & $\mathbf{3 7 , 1} \pm \mathbf{8 , 7}$ \\
\hline Jornada Laboral & $8 \mathrm{~h}$ & $51(33,3 \%)$ \\
& $9 \mathrm{~h}$ & $39(25,5 \%)$ \\
& $10 \mathrm{~h}$ & $63(41,2 \%)$ \\
Actividad Laboral & & \\
& Ordenadores & $85(55,6 \%)$ \\
& Recepción & $53(34,6 \%)$ \\
Mediana del tiempo laboral /min - máx. meses & Archivo y almacén & $15(9,8 \%)$ \\
& & \\
Presencia de Hiperlaxitud Articular & 144 & $3-420$ \\
& & \\
& & \\
& No & $68(57,5 \%)$ \\
\end{tabular}


se encontró que la mediana del tiempo laborando es de 144 meses, con un mínimo de 3 meses y un máximo de 420 meses (tabla 1).

Con relación a la frecuencia de HA en mujeres, se observa que la tendencia va disminuyendo en edades más adultas $(\mathrm{p}<0,05)$ (tabla 2$)$. La prevalencia de HA en mujeres de 20 a 30 años es 3,3 veces más en comparación con las mujeres mayores de 40 años y la prevalencia de HA en mujeres de 30 a 40 años es 2,7 veces más en comparación en persona mayores de 40 años (tabla 3 ).

Respecto a la jornada laboral, se aprecia que las mujeres que laboran 8 horas diarias el $39.2 \%$ presenta
HA, las que laboran 9 horas el 51,3\% presenta HA y de las que laboran 10 horas el 39,7\% presenta HA. Del total de las que presentaron HA, el $38.5 \%$ corresponde a las que laboraban 10 horas (tabla 4). En el caso de actividad laboral, el 43,5\% que se desempeñaban como usuaria de ordenadores presentó HA; el 47,2\% que laboraban en la recepción presentó HA, y el 20\% de las que laboraban en archivo y almacén presentaron HA (tabla 5), del total que presentaron HA, el 56,9\% se desempeñaban como usuarias de ordenadores.

De las zonas evaluadas se observa que la extensión pasiva del dedo meñique derecho presenta mayor frecuencia de HA siendo el 54,9\% (IC 95\%: 46,93$62,88)$, seguido de extensión pasiva de meñique

Tabla 2. Hiperlaxitud articular según edad

\begin{tabular}{lccl}
\hline Edad (años) & \multicolumn{2}{c}{ Hiperlaxitud Articular (HA) } & \multirow{2}{*}{ p } \\
\cline { 2 - 3 } & No & Si & \\
\hline Mediana/ min. - máx. & $40,5(21-55)$ & $32(20-50)$ & \\
$20-<30$ & $13(34,2 \%)$ & $25(65,8 \%)$ & $<$ \\
$30-<40$ & $23(46,0 \%)$ & $27(54,0 \%)$ & 0,05 \\
$\geq 40$ & $52(80,0 \%)$ & $13(20,0 \%)$ & \\
\hline
\end{tabular}

Tabla 3. Prevalencia HA según edad

\begin{tabular}{llr}
\hline Hiperlaxitud Articular & RP & Valor de p \\
\hline Edad & & \\
$20-30$ & 3,29 & 0 \\
$30-40$ & 2,7 & 0 \\
$\geq 40$ & & \\
\hline
\end{tabular}

Tabla 4. Presencia de HA y jornada laboral

\begin{tabular}{cc}
\hline Jornada Laboral & Si \\
\hline $8 \mathrm{~h}$ & $20(30,8 \%)$ \\
$9 \mathrm{~h}$ & $20(30,8 \%)$ \\
$10 \mathrm{~h}$ & $25(38,5 \%)$ \\
Total & 65 \\
\hline
\end{tabular}

Tabla 5. Hiperlaxitud articular actividad laboral

\begin{tabular}{lccc}
\hline \multirow{2}{*}{ Actividad Laboral } & \multicolumn{2}{c}{ Hiperlaxitud Articular (HA) } & \multirow{2}{*}{$\mathbf{p}$} \\
\cline { 2 - 3 } & No & Si & \\
\hline Ordenadores & $48(56,5 \%)$ & $37(43,5 \%)$ & \\
Recepción & $28(52,8 \%)$ & $25(47,2 \%)$ & 0,185 \\
Archivo & $12(80 \%)$ & $3(20 \%)$ & \\
\hline
\end{tabular}


Tabla 6. Hiperlaxitud articular según puntaje

\begin{tabular}{ccc}
\hline Puntaje & Frecuencia & $\mathbf{\%}$ \\
\hline 4 & 7 & 10,77 \\
5 & 20 & 30,77 \\
6 & 20 & 30,77 \\
7 & 13 & 20 \\
8 & 5 & 7,69 \\
Total & 65 & 100 \\
\hline
\end{tabular}

izquierdo con el 45,1\% (IC 95\%: 37.1253,07) y extensión de codo derecho con el 45,1\% (IC 95\%: $37,12-53,07)$. Del total de hiperlaxas el $30.7 \%$ obtuvo un puntaje total de 5 en el test de Beighton, el mismo resultado se obtuvo para el puntaje de 6 , siendo estos los máximos porcentajes obtenidos (tabla 6). De acuerdo a las que obtuvieron puntaje positivo en el test de Beighton según las zonas que evalúa y la actividad laboral se obtuvo que, las que laboraban en ordenadores el $69,4 \%$ presentó mayor hiperlaxitud en rodilla derecha seguido de rodilla izquierda con el $64,4 \%$; las que laboraban en recepción el $42,8 \%$ presentó hiperlaxitud en meñique derecho seguido del 40,5\% en pulgar izquierdo; y las que laboraban en archivo y almacén el 14,4\% presentó hiperlaxitud en meñique izquierdo seguido del $11,5 \%$ en codo izquierdo.

\section{DISCUSIÓN}

En nuestro estudio la frecuencia de HA en mujeres fue de $42.5 \%$, siendo un porcentaje diferente a los realizados por Farro y cols, Barrantes y cols, Russek y Errico $(11,12,13)$. Esta diferencia en los resultados puede deberse al tamaño de muestra utilizada, sin embargo, el intervalo de confianza obtenido al ser relativamente pequeño nos otorga confiabilidad en los resultados. Además, esta diferencia también se puede deber a las características de las poblaciones estudiadas tales como edad, antecedentes étnicos y sexo; ya que nuestro estudio consideró un rango de edad productiva en el ámbito laboral entre 20 y 65 años, mientras que otros estudios consideraron edades hasta los 45 años. Además, nuestro estudio solo consideró mujeres, que se desempeñan en áreas administrativas realizando trabajos de carga estática manteniendo posturas forzadas y ejecutando actividades repetitivas.

Por otro lado, nuestros resultados evidenciaron una prevalencia de HA en mujeres de 20 a 30 años del $65,8 \%$, mientras que en mujeres mayores de 40 años es de $20 \%$. En este estudio se concluye que la prevalencia de hiperlaxitud articular en mujeres de 20 a 30 años es 3,3 veces más que en mujeres mayores de 40 años y la prevalencia de HA en mujeres de 30 a 40 años es 2,7 veces más que en mujeres mayores de 40 años; lo cual, se debería a la influencia hormonal especialmente de la relaxina, ya que a medida que aumentan los años los niveles de esta hormona decaen y por tanto la presencia de hiperlaxitud es menor $(6,10)$.

Respecto a la jornada laboral, de las que presentaron HA el 38,5\% labora 10 horas diarias, por lo que al hallarse una frecuencia de HA elevada y una larga jornada de trabajo, las hace más vulnerable a padecer lesiones musculo esqueléticas por lo que Sáenz y cols, sugieren que se establezca un máximo de horas consecutivas de labor entre 5 y 7 horas ya que existe sobreexposición a los riesgos laborales debido a jornadas sucesivas de trabajos mayor a 6 horas diarias (14). Riascos por su lado, refiere que las posturas prolongadas van ligadas a la presencia de sintomatología musculo esqueléticas, ya que aproximadamente el $45 \%$ de los administrativos permanecen en una postura por 6 a 8 horas diarias (15).

Las principales actividades laborales realizadas por el personal administrativo de nuestro estudio se agrupan, en archivo y almacén, usuarios de ordenadores y personal de recepción, que al relacionarlas con HA no establece relación significativa ( $p>0,01)$; a pesar de esto, la frecuencia más alta obtenida con HA (56,9\%) corresponde al personal administrativo usuario de ordenadores. De acuerdo con las zonas, los segmentos evaluados con mayor porcentaje de Hiperlaxitud articular son el codo derecho (45,1\%), meñique derecho $(54,9 \%)$ y meñique izquierdo $(45,1 \%)$, siendo estas estructuras las más empleadas por los usuarios de ordenadores. El estudio de Sáenz también refiere que el $26,7 \%$ de trabajadoras administrativas presentaron dolor de mano en los últimos 6 meses, además el $16,7 \%$ de ellas refirió que este padecimiento les impide realizar sus actividades con normalidad (14). Finalmente, en base a los criterios de Beighton se halló que el $30.7 \%$ de las mujeres obtuvieron 5 y 6 de puntaje, siendo un porcentaje importante en esta población y consideradas de alto riesgo para el padecimiento o agravamiento de lesiones musculo esqueléticas.

\section{Declaración de conflicto de interés}

Los autores del proyecto de investigación declaramos no tener ningún tipo de conflicto de 
intereses, ni ninguna relación económica, personal, política, interés financiero ni académico que pueda influir en nuestro juicio. También, afirmamos no haber recibido ningún tipo de beneficio monetario, bienes ni subsidios de alguna fuente que pudiera tener interés en los resultados de esta investigación. Asimismo, las personas o instituciones que hayan participado en la recolección y análisis de la información han sido identificadas en los agradecimientos y han aceptado dicha mención; al tratarse de un estudio con seres humanos, manifestamos que se obtuvo la aprobación del Comité Institucional de Ética en Investigación de la Universidad Peruana Cayetano Heredia.

\section{REFERENCIAS BIBLIOGRAFICAS}

1. Pantoja L, Díez C, Duckens A. Sindrome de EhlersDanlos hiperlaxo: ¿Hipermovilidad benigna? Reumatol Clin. 2014; 10(3): 189-190.

2. Schubert E. Pain, balance, activity, and participation in children with hypermobility syndrome. Pediatr Phys Ther. 2012; 24 (4): 339-44.

3. Gonzales R, Oliva Y. El síndrome de hiperlaxitud articular, un enfoque clínico epidemiologico en Minas de Matahambre. Rev Ciencias Médicas. 2014; 18(1): 45-46.

4. Bravo J. Significado e importancia de estudiar a las personas con Hiperlaxitud Articular. Rev Chil Reum. 2008; 24(1): 4-5.

5. Haro M, Morante M, Lillos S. Síndrome de hiperlaxitud articular benigno en el niño. Rev. Med. Clin Condes. 2014; 25(2): 255-264.

6. Guitart J. Análisis sobre la laxitud ligamentosa en función de los niveles de relaxina, la inestabilidad de tobillo que se deriva y su tratamiento en consulta podológica. Tesis de Grado. Barcelona: Universitat de Barcelona2014.

7. Sánchez A. Encuesta Nacional a egresados y universitarios 2014. Lima: Instituto nacional de estadística e informática; 2015.
8. Strazdins L, Bammer G. Women, work and musculoskeletal health. Soc Sci Med. 2004; 58:9971005.

9. Ministerio de trabajo y promoción de empleo. Resolución Ministerial $\mathrm{N}^{\circ}$ 375-2008-TR. Norma Básica de Ergonomía y de Procedimiento de Evaluación de Riesgo Disergonómico. Aprobado el 30 de noviembre del 2008. Perú. Lima; Ministerio de trabajo y promoción de empleo 2008.

10. Beighton P, Solomon L, Soskolne CL. Articular mobility in an African population. Ann Rheum Dis. 1973; 32(5): 413-418.

11. Farro-Uceda L, Tapia Egoavil R. Relación ente hiperlaxitud articular, dismetría de miembros inferiores y control postural con los trastornos posturales. Rev Med Hered. 2016; 27(4):216-222. DOI: 10.20453/rmh.v27i4.2990

12. Barrantes T. Síndrome benigno de hipermovilidad articular: análisis de la prevalencia, rasgos somato métricos y asociaciones clínicas frecuentes. CIMEL. 2001; 6: 39-43.

13. Russek L, Errico M. Prevalence, injury rate and, symptom frequency in generalized joint laxity and joint hypermobility syndrome in a "healthy" college population. Clin Rheumatol. 2016;35(4):1029-39. doi: 10.1007/s10067-015-2951-9

14. Sáenz M, Rodriguez R, Marulanda N, Gomnez M. Morbilidad sentida osteomuscular y riesgo por carga física en trabajadores de servicios administrativos. RSCO. 2016; 6(1). DOI: 10.18041/2322-634X/ rcso.1.2016.4881

15. Riascos D, Martinez L, Eraso N, Rodriguez Y. Sintomatología musculo- esquelética, posturas y posiciones corporales en el personal administrativo del IDSN. Bogota: UNIMAR.

Recibido: $12 / 08 / 2021$

Aceptado: 28/11/2021 